\title{
Line Segmentation and Orientation Algorithm for Automatic Bengali License Plate Localization and Recognition
}

\author{
Md. Rokibul Haque \\ B.Sc. Student \\ Sylhet Engineering College \\ Sylhet-3100
}

\author{
Saddam Hossain \\ B.Sc. Student \\ Sylhet Engineering College \\ Sylhet-3100
}

\author{
Sagor Roy \\ B.Sc. Student \\ Sylhet Engineering College \\ Sylhet-3100
}

\author{
Nashid Alam \\ Assistant Professor, Department of CSE \\ Sylhet Agriculture University \\ Sylhet-3100
}

\author{
M. Jahirul Islam, PhD PEng. \\ Professor, Department of CSE \\ Shahjalal University of Science and Technology \\ Sylhet-3100
}

\begin{abstract}
Automatic License Plate Recognition (ALPR) is an image processing technology which used to identify vehicles by their license plate. In this paper, an ALPR system is developed where adaptive resize and crop are used for better efficiency. For highlighting the license plate region image morphology, horizontal and vertical extraction has been used. Line Segmentation and Orientation (LSO) algorithm is proposed for segmentation which is also effective in efficient searching for signs of the license plate and finally template matching has been used for recognition. The proposed method works successfully with the recognition rate of $84.87 \%$. Moreover, some license plates with different fonts of writings have been given true positive results which are not issued by the government. So, the proposed method will be helpful not only in license plate recognition but also in other field of Bangla optical character recognition.
\end{abstract}

\section{Keywords}

ALPR; Image Morphology; CC; LSO Algorithm; Sign House Database; Template Matching;

\section{INTRODUCTION}

Car license plate is a metal or plastic built rectangular shape which is a part of the vehicle. It is fixed with both front and rear side of every vehicle. A car license plate generally contains words, letter and digits which give the identity of the vehicle. The license plate of the vehicle is given by BRTA [1] in Bangladesh. In Bangladesh, there is very less implementation of ALPR system. But there are many field of that system like speed limit and traffic rules check, parking lot management, automatic toll collection system, stolen vehicle search etc. Moreover, traffic is increasing day by day. So, if the traffic related system is handled using ALPR system it would be the most effective solution for all those problems.

Amin et. al. [2] developed an ALPR system of Bangladeshi vehicles where Sobel edge detection method [3] has been used for detecting the edges. Hough transformation [4] which detects lines and curve is used to locate and highlighting the license plate region. For localizing the license plate region image matrix has been created and using this rectangular shape of the license plate has been searched. More over Otsu threshold [5] is used for processing the license plate. Finally, Tesseract [6] is used for character recognition and the recognition rate of the method was $62 \%$. But before recognition there need to segment the characters of the license plate. In this method, there is no discussion about segmentation of the characters.

Another method proposed by Ghosh et. al. [7] where vertical and Sobel edge detection has been used for detecting the edges. After that image morphology [8] and aspect ratio have been used to localize the license plate region. For straighten the license plate image skew of license plate has been detected. After that both lines and characters of license plate have been segmented. For line segmentation of the writings of license plate horizontal scanning has been used and removing the 'matra' of the words for word segmentation. Finally, for feature extraction and recognition Freeman chain code [9] and neural network [10] have been used respectively. This method worked successfully with the accuracy rate of $80 \%$. But in this method segmentation of the words of license plate by removing 'matra' of the words changes the shape of the characters which may gives wrong result in many occasions. Moreover, horizontal scanning for line segmentation may give wrong result due to the border of the license plate.

Mashuk et. al. [11] discussed a method and it is worked in three stages which are detection, segmentation and recognition. For detection and crop the license plate pixel by pixel search has been used. Connected Component (CC) analysis is used for the segmentation of the digits of the cropped license plate. For the recognition of the license plate neural network has been used. But this method only extracted the digits from the license plate. Moreover, pixel by pixel search is not an efficient process to detect the license plate. 


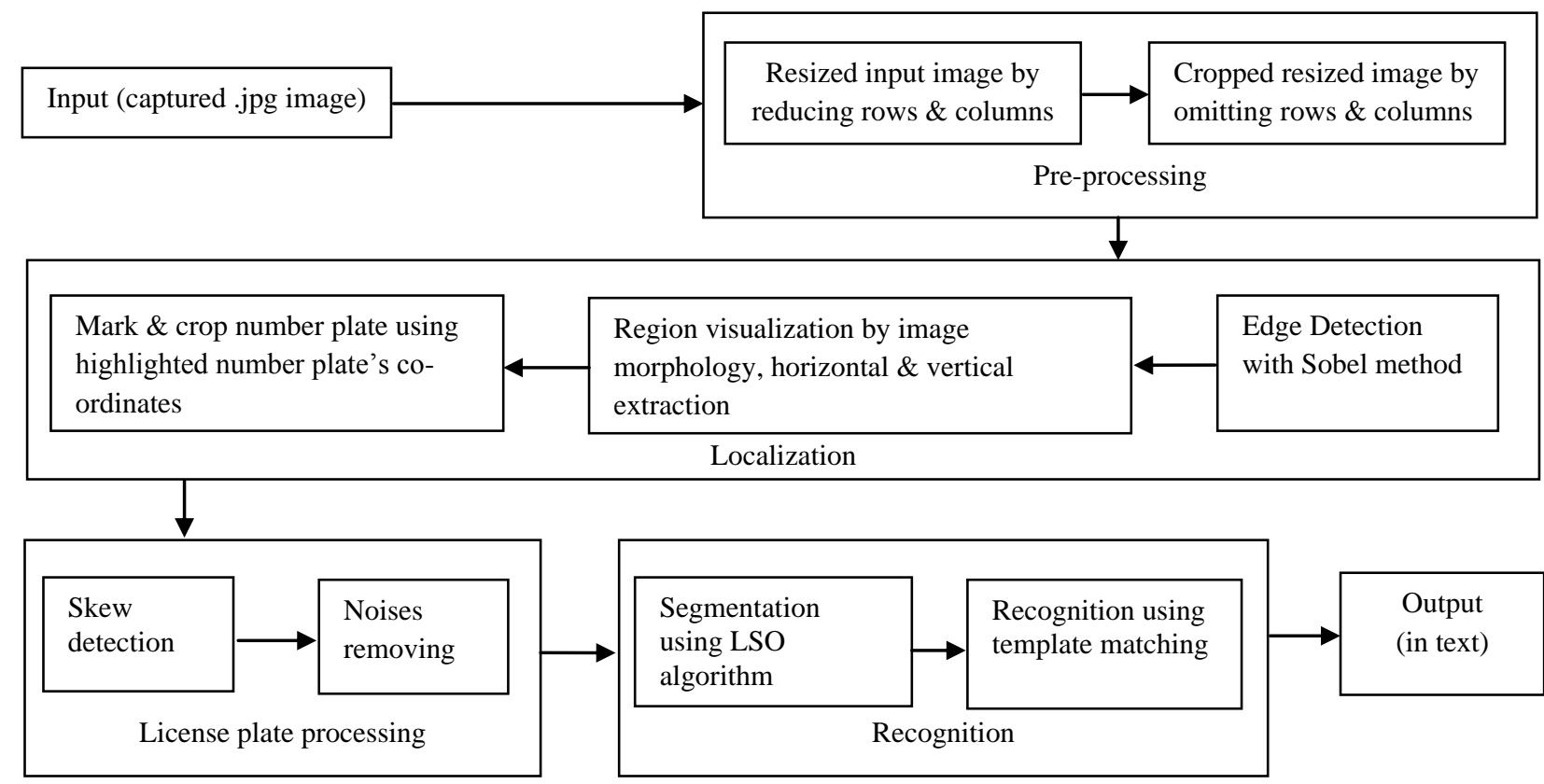

Fig 1: Overall working procedure

\section{METHODS \& MATERIALS}

A method has been proposed which is mainly divided into four parts. These four parts are pre-processing, localization, license plate processing and recognition. Automatic resize and crop are the part of the preprocessing. Edge detection, image morphology, horizontal and vertical extraction are used for highlighting the license plate region. Skew detection and noise removal are the part of license plate processing. Finally, segmentation using LSO algorithm and template matching [12] are used for the recognition system. The overall proposed method is shown in fig 1 .

\subsection{Proposed Algorithm}

In this method captured jpg type image has been used as input image. The pseudo code of the proposed algorithm is given below:

- $\quad$ Resize the input image by reducing the total number of rows and columns into eight hundred and twelve hundred respectively.

- Crop resized image by omitting two hundred rows from top and two hundred columns from both left and right part of the resized image.

- Convert the cropped image into gray scale image.

- Detect edges using Sobel edge detector.

- Highlight the license plate region using image morphology, horizontal \& vertical extraction.

- Localize and crop the number plate from cropped image using the co-ordinates of highlighted license plate region.

- Convert the cropped license plate image into grayscale image.

- Convert the gray scale image into binary image using Otsu method.
- Find modified license plate image using image complementation to Otsu image.

- Straighten license plate image using the measurement of skew.

- Remove noises by cropping boundaries and removing small connected components.

- Segment and oriented the words and the characters of the noise removed license plate image using LSO algorithm.

- Recognize the words and characters using image correlation with Sign House database's image.

- Show the output in text.

\subsection{Pre-processing}

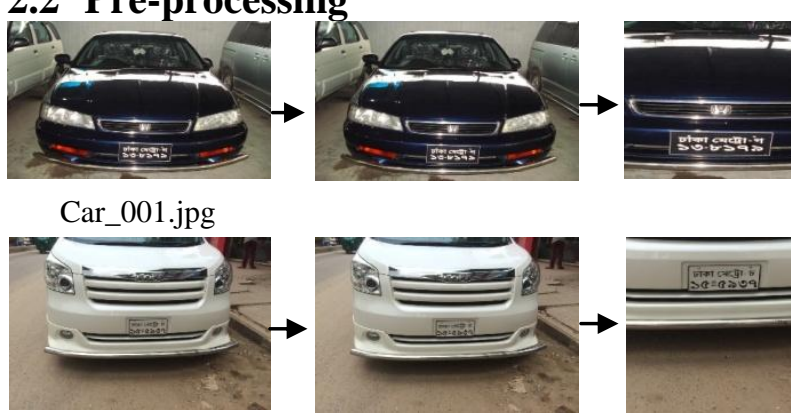

Noah_01.jpg

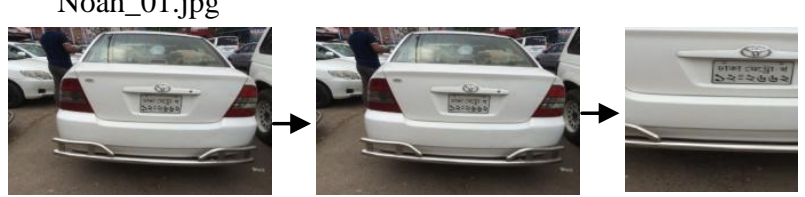

Car_002.jpg

a) Captured image b) Resized image by c) Cropped reducing rows \& image by columns omitting rows

\& columns

Fig 2: Pre-processing by resizing and cropping 
In order to simplify process firstly the captured input image which is shown in fig 2(a) is resized into the resolution of 800 x 1200 by using two elements of position vector expected rows (800) and columns (1200). The resized image is shown in fig 2(b). Generally, almost every vehicle contains license plate middle lower part of the body both at front and rear side.
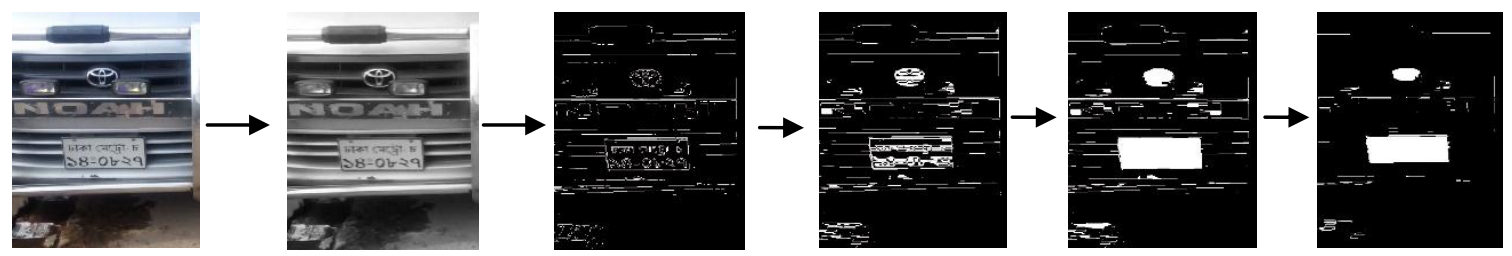

Noah_02.jpg
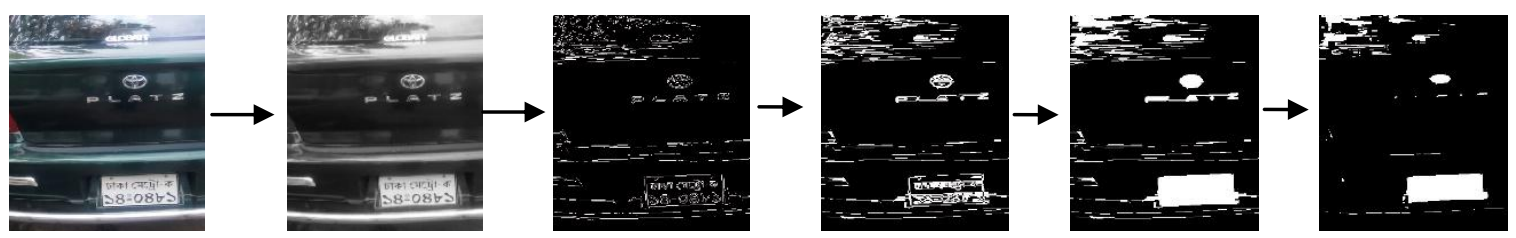

Micro_01.jag
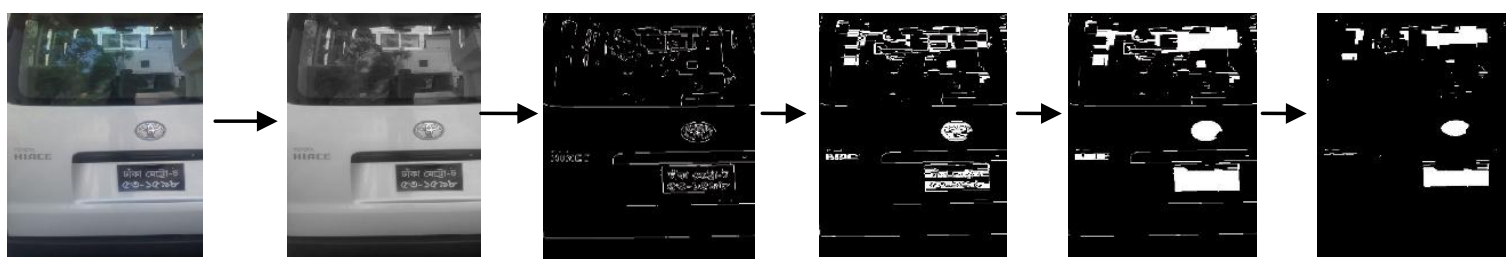

Micro_04.jpg
a) Cropped image
b) Gray image
c) Sobel edged image

\begin{abstract}
d) Horizontally extracted image
\end{abstract}

f) Vertically extracted image e)

Morphological analyzed image

Fig 3: Highlighting process of license plate region

\subsection{Localization}

In this phase, firstly it is needed to highlight the license plate region. Therefore, the cropped image shown in fig 3(a) is converted into gray scale image and is shown in fig 3(b). After that Sobel edge detector has been used in gray scale image to highlight the edges which is shown in fig 3(c). Two $3 \times 3$ kernels which are convolved with the grayscale image to calculate approximations of the derivatives are used by Sobel detection method one for horizontal changes, and one for vertical changes. Now for highlighting the license plate region from Sobel edged image horizontal extraction is applied. In horizontal extraction each pixel value of Sobel edged image has been checked horizontally and makes the pixel values from 0 to 1 within next twenty-five pixels after getting a pixel value 1 . If any pixel value is 1 within that twenty-five pixels, process will be stopped and it will be continued from the next pixel. As a result, horizontally extracted image has been found and that is shown in fig 3(d). After that image morphology has been used to fill the holes of the horizontally extracted image and found a morphological analyzed image which is shown in fig 3(e). To avoid unexpected edges connected with highlighted license plate region, the pixel values of morphological analyzed image have been checked vertically and makes the pixel's values 0 to 1 if there are at least fifteen pixel values of 1 are not together. Finally, vertically extracted image has been found which is shown in fig 3(f). For determining the exact $\mathrm{CC}$ of highlighting license plate region from vertically extracted image three decision constants have been used. First one is the number of rows of the CC must less than seven tenth of the column, second is that the number of columns must less than six hundred and greater than one hundred and the third one is that the $\mathrm{CC}$ must has the greater white pixel ratio. After finding the exact $\mathrm{CC}$ from vertically extracted image using it's coordinates license plate is localized and cropped from croped image which is shown in fig 4(b). The localized and cropped license plate images are shown in fig 4(c) and fig 4(d) respectively. 

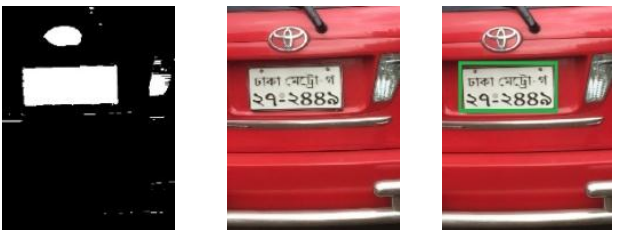

जांका बस्द्धा भा

২৭-২৪৪৯

Jeep_02.jpg
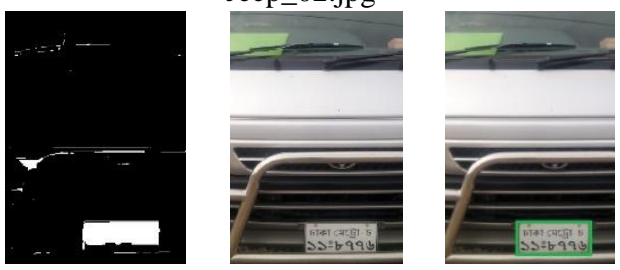

Micro_06.

jpg
c) Localized
d) Cropped license plate image using three constants

Fig 4: License plate marking and cropping process

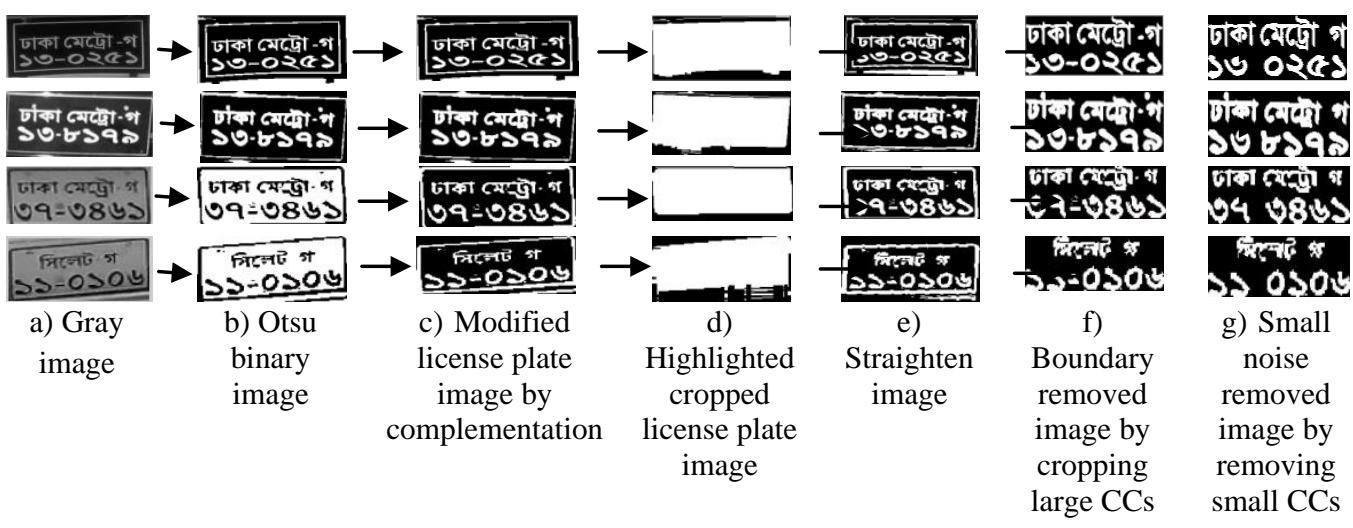

Fig 5: License plate processing

\subsection{License Plate Processing}

In order to simplify the processing cropped license plate image has been converted to gray scale image which is shown in fig 5(a). After that the gray image is converted into binary image using Otsu threshold method and that is shown in fig 5(b). In this method, it deals with two set of pixels' variance which is background and foreground. A threshold value was searched that minimizes the intra-class variance (the variance within the class), defined as a weighted sum of variances of the two classes:

$\sigma_{\mathrm{w}}^{2}(t)=w_{0}(t) \sigma_{0}^{2}(t)+w_{1}(t) \sigma_{1}^{2}(t)$

Weights $w_{0,1}$ are the probabilities of the two classes separated by a threshold $t$ and $\sigma_{0,1}^{2}$ are variances of these two classes. The class probability $w_{0,1}(t)$ is completed from $\mathrm{L}$ histograms:

$w_{0}(t)=\sum_{i=0}^{t-1} p(i)$

$w_{1}(t)=\sum_{i=t}^{L-1} p(i)$
In this method minimizing the intra-class variance is the same as maximizing inter-class variance:

$$
\begin{aligned}
\sigma_{\mathrm{b}}^{2}(t) & =\sigma^{2}-\sigma_{\mathrm{w}}^{2}(t) \\
& =w_{0}\left(\mu_{0}-\mu_{\mathrm{T}}\right)^{2}+w_{1}\left(\mu_{1}-\mu_{\mathrm{T}}\right)^{2} \\
& =w_{0}(t) w_{1}(t)\left[\mu_{0}(t)-\mu_{1}(t)\right]^{2}
\end{aligned}
$$

Which is expressed in terms of class probabilities $w$ and class mean $\mu$.While the class mean $\mu_{0,1, T}(t)$ is:

$$
\begin{aligned}
& \mu_{0}(t)=\sum_{i=0}^{t-1} i p(i) / w_{0} \\
& \mu_{1}(t)=\sum_{i=t}^{L-1} i p(i) / w_{1} \\
& \mu_{T}=\sum_{i=0}^{L-1} i p(i) \\
& \text { After Otsu threshold two different types of background of } \\
& \text { Otsu image can be found. But for the further process there } \\
& \text { always need to white writings in black background. In that } \\
& \text { case firstly calculate the number of white and black pixel of } \\
& \text { Otsu image individually. The greater number of pixel amount } \\
& \text { represents the background color. So, if the number of white }
\end{aligned}
$$


pixel is greater than the number of black pixel than complement the Otsu image other ways there is no need to complementation. Finally, modified license plate image has been found which is shown in fig 5(c).

\subsubsection{Skew Detection}

Sometime the processed license plate may be skewed. So, for detecting the skew orientation of the $\mathrm{CC}$ of highlighted cropped license plate image which is shown in fig 5(d) has been measured and using that measurement the modified license plate image has been straightened which is shown in fig 5(e).

\subsubsection{Noise Elimination}

Boundaries and unexpected small CCs are the noises of a license plate. In order to remove boundaries, the coordinates of the CCs of straighten image which total number of rows and columns are less than one sixth of rows and columns of straighten image respectively are calculated. From those CCs coordinates top left most and bottom right most coordinates are found. These two co-ordinates are used to crop the straighten image to get boundary removed image which is shown in fig 5(f). Now CCs of boundary removed image which total number of rows and columns are less than six tenth of the total number of rows and columns of boundary removed image has been removed. Finally, small noise removed image which is shown in fig $5(\mathrm{~g})$ has been found.

\subsection{Recognition}

Generally, there are two lines of CC in the license plate. So, in order to segment the lines and oriented the CCs orderly LSO algorithm is applied into the small noise removed image which is shown in fig 6(a). Segmentation result using LSO algorithm is shown in fig $6(\mathrm{~b})$ and the pseudo code of the algorithm is given below:

1. Calculate the average of top and bottom row of a random connected component from small noise removed image and save the connected component in an array.

2. Check and save rest connected components one by one in the array along with the chosen random connected component if their number of top row is less and number of bottom row is greater than the chosen random connected component's average row's value. Other ways save the connected component another way.

3. Rearrange the connected components of both arrays according to the ascending order of their top row number in the small noise removed image.

4. According to the number of top row in small noise removed image of first connected component of both arrays first and second line has been chosen.
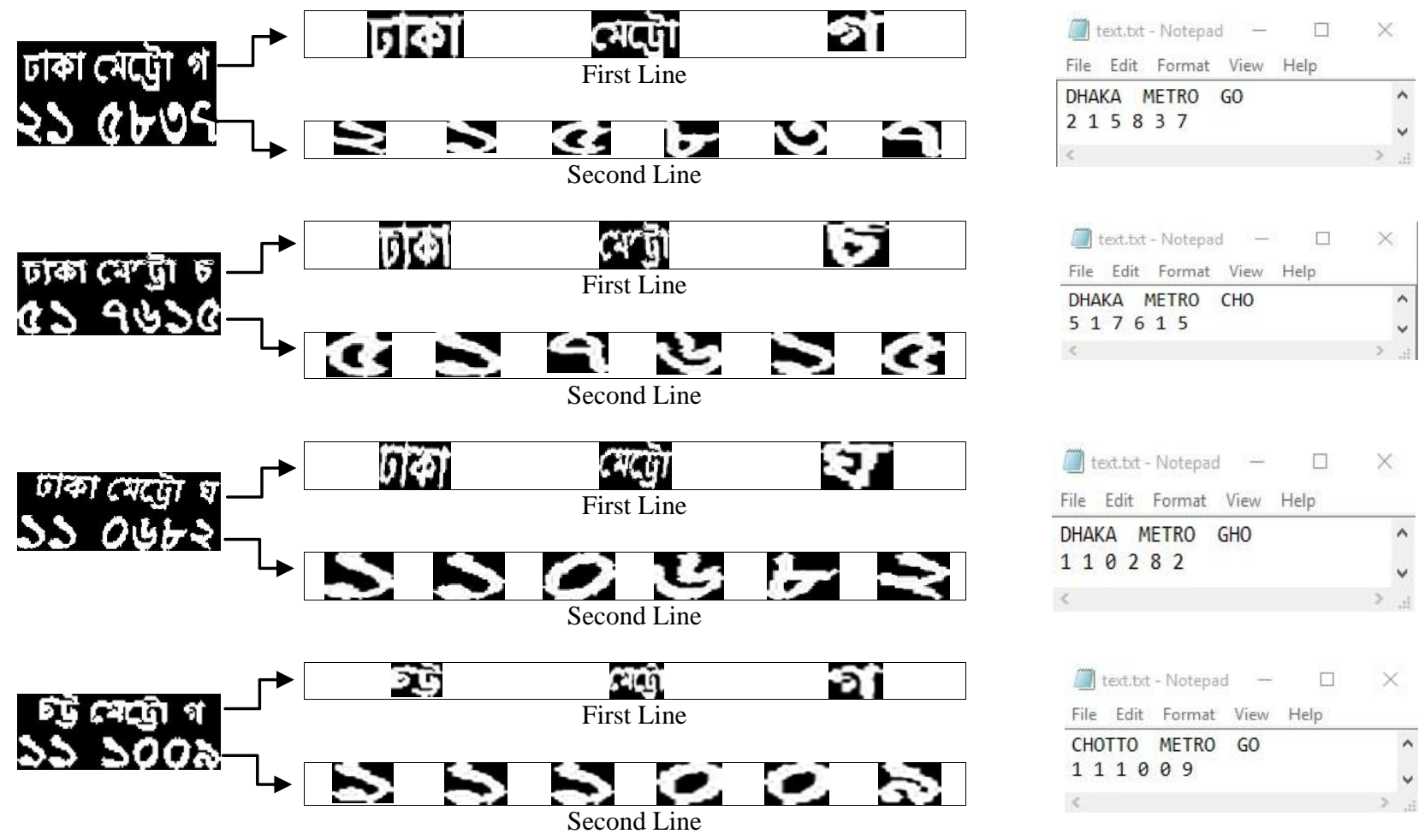

a) Small noise removed b) Writing segmentation using LSO algorithm image

Fig 6: Segmentation with LSO algorithm and recognition result from template matching
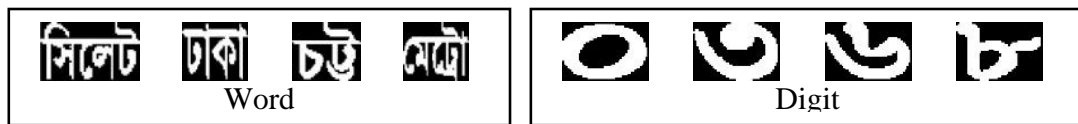

Fig 7: Example of Sign House database

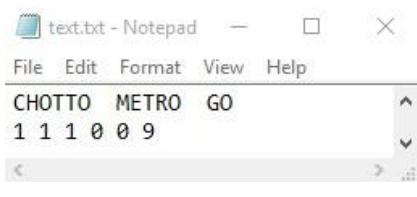

c) Recognition result using template matching in text 
For recognition, template matching has been used. So, a template database named 'Sign House' has been created where the sign images of license plate are stored. There are three types of signs (word, alphabet and digit) have been stored in that database. Examples of 'Sign House' are shown in fig 7. Generally, first line of license plate contains one or two word and one alphabet and the alphabets is the last sign of first line. So, last image of first line have been matched with alphabets and others images of first line are matched with words of 'Sign House'. Second line contains only digits. So, all the images contained by second line have been matched with digits of 'Sign House'. Among the highest percentage of matching result decision has been made and shows the recognition result in text which is shown in fig 6(c). In this matching image correlation [13] has been used. For image correlation operation between two images there must be same size of the both images. So, before using correlation all images of first and second line resized into $32 \times 48$ sizes because all the images of 'Sign House' are stored in that (32x48) size.

\section{RESULT ANALYSIS}

The license plates of the captured images have been categorized with three different types which are standard license plate, wrong font license plate and old number plate. Standard license plates are those license plates which are issued by the government, wrong font license plate means those license plates which are contained different font of writings in the license plate and not issued by government and old license plates means license plate with dirt, dust, unclear writings and bumper in front of writings. Some examples of those three types of number plate are shown in fig 8 . There are 119 vehicle images have been captured for testing this proposed method where standard license plate is contained by 93 images, wrong font license plate is contained by 15 images and old license plate is contained by 11 images.
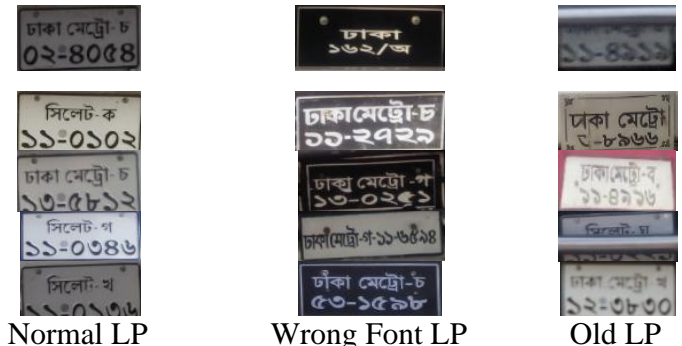

Fig 8: Different types of license plate

Among 93 standard license plates 92 have been localized, 90 have been extracted and 87 have been recognized. From 15 wrong font license plates 14 have been localized, 11 have been extracted and 9 have been recognized and from 11 old license plates 8 have been localized, 6 have been extracted and 5 has been recognized. The localization, extraction and recognition rate of these three types of number plate have been represented with the bar chart which is shown in fig 9 .

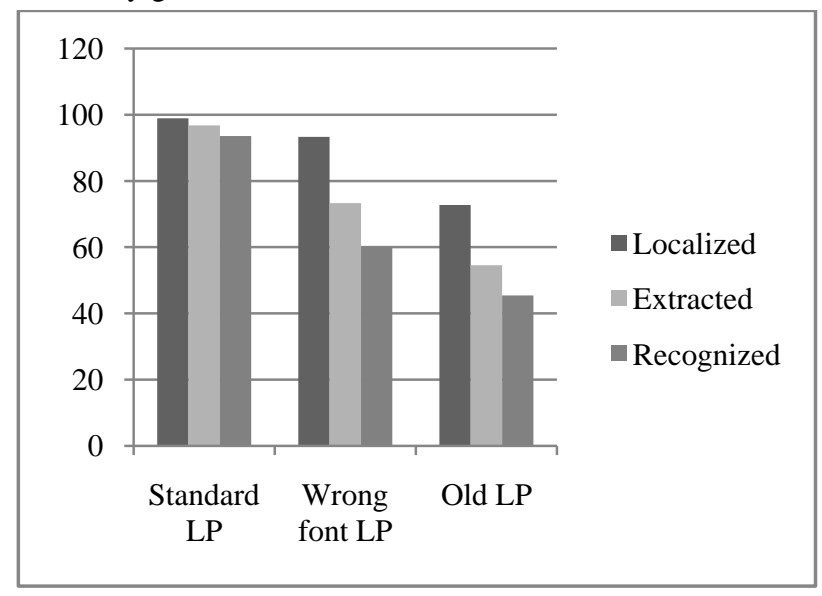

Fig 9: Accuracy rate in different types of license plate

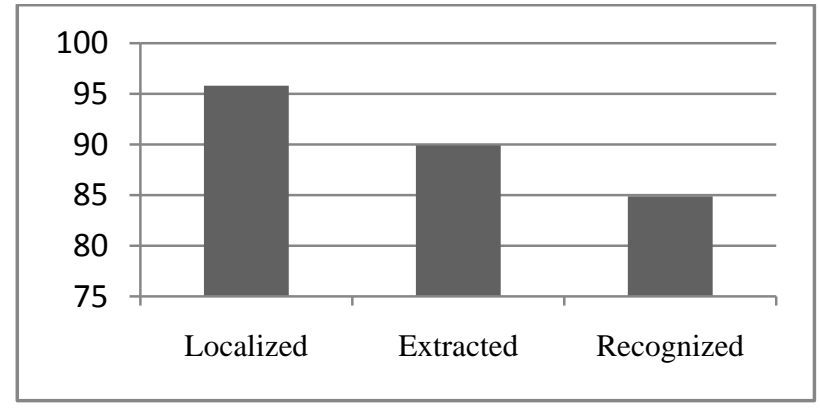

Fig 10: Overall accuracy rate

Now from total 119 vehicle's images 114 license plates are localized by the proposed method. Among the 114 localized license plates, writings of the license plate are extracted from 107 images and finally 101 have been recognized. The overall rate of localization, extraction and recognition is shown in fig 10 using bar chart. Comparative analysis with some other method and result analysis of the proposed method are shown in table- 1 and table- 2 respectively. 
Table 1 Comparative analysis of Bangladeshi car license plate detection

\begin{tabular}{|c|c|c|c|c|}
\hline Author Reference & Approach & Country & Localization Rate & Recognition Rate \\
\hline $\begin{array}{l}\text { Deb, K., Chae, H.U., Jo, K.H. } \\
\text { (2009) [14] }\end{array}$ & $\begin{array}{l}\text { HSI color model and } \\
\text { histogram }\end{array}$ & South Korea & Not discussed & $82.5 \%$ \\
\hline $\begin{array}{c}\text { Saha, S., Basu, S., Nasipuri, M. } \\
\text { (2011) [15] }\end{array}$ & Template matching & India & $90 \%$ & $80.20 \%$ \\
\hline $\begin{array}{l}\text { Ghosh, A.K., Sharma, S.K.D., } \\
\text { Islam, M.N., Biswas, S., Akter, S. } \\
\text { (2011) [7] }\end{array}$ & Neural Network & Bangladesh & $84 \%$ & $80 \%$ \\
\hline $\begin{array}{l}\text { Shidore, M. M., Narote, S. P. } \\
\text { (2011) [16] }\end{array}$ & $\begin{array}{l}\text { Support Vector machine } \\
\text { (SVM) }\end{array}$ & India & $85 \%$ & $89.84 \%$ \\
\hline $\begin{array}{l}\text { Amin, M.R., Mohammad, N., Md. } \\
\text { Bikas, M.A.N. (2014) [2] }\end{array}$ & Tesseract 3.0 & Bangladesh & $88 \%$ & $62 \%$ \\
\hline Proposed Method & $\begin{array}{l}\text { Edge Detection and } \\
\text { Template Matching }\end{array}$ & Bangladesh & $95.8 \%$ & $84.87 \%$ \\
\hline
\end{tabular}

From the bar chart of fig 9 it is cleared that the accuracy rate of recognition is higher in the standard type of license plate. So, if all the vehicles use the standard license plate which is issued by the government the accuracy rate of the proposed method will be increased. However, some license plate of different font of writings and some old license plates are recognized by the proposed method which is encouraging.

Table 2 Result analysis of the proposed method

\begin{tabular}{|c|c|}
\hline & Rate (\%) \\
\hline True Positive (TP) & 84.87 \\
\hline True Negative (TN) & 4.21 \\
\hline False Positive (FP) & 3.36 \\
\hline False Negative (FN) & 7.56 \\
\hline
\end{tabular}

\section{CONCLUSION}

In this paper a method of automatic Bengali car license plate has been proposed which works successfully with the accuracy rate of $84.87 \%$. But this accuracy rate will be increased if all the vehicles use the license plate issued by the government. Image processing toolbox in Matlab- R2014a is used to implement this method. For capturing the test images iPhone $5 \mathrm{~s} \&$ Walton primo Rx2 devices are used. Adaptive crop and resize have been used in this method which makes the process easier. Moreover, horizontal and vertical extraction is helped to localize the license plate accurately. LSO algorithm has been proposed which helps the segmentation and matching of the writings very accurately and efficiently. The method has been based on static image. So, in future it would be better if this method is implemented from real time video footage and at the same time, obviously increasing the accuracy is a big concern.

\section{REFERENCES}

[1] http://www.brta.gov.bd/services/digital-numberplate.html

[2] Amin, M.R., Mohammad, N. and Bikas, M.A.N. (2014) 'An Automatic Number Plate Recognition of Bangladeshi Vehicles', International Journal of Computer Applications (0975 - 8887)), vol. 93, No15.

[3] Sobel, I. and Feldman, G. (1968) 'A 3x3 Isotropic Gradient Operator for Image Processing', Stanford Artificial Intelligence Project (SAIL).

[4] Duda, R.O. and Hart, P.E. (1972) 'Use the Hough Transformation to detect the lines and curves in the picture', Comm. ACM, vol 15, No.pp. 11-15.

[5] Otsu, N. (1979) 'A Threshold Selection Method from Gray- Level Histograms', IEEE Transactions on Systems, Man and Cybernetics, vol. 9, No 1, pp. 62-66.

[6] Chowdhury, A., Foysal, A. and Islam, S. (2016) 'Bangla Character Recognition for Android Devices', International Journal of Computer Applications (0975 8887) Volume 136- No.11.

[7] Ghosh, A.K., Sharma, S.K.D., Islam, M.N., Biswas, S. and Akter, S. (2011) 'Automatic License Plate Recognition (ALPR) for Bangladeshi Vehicles', Global Journal of Computer Science and Technology, vol. 11, Issue 21, Version 1.0.

[8] Sreedhar, K. and Panlal,B. (2012) 'Enhancement of images using morphological transformations', International Journal of Computer Science \& Information Technology (IJCSIT) Vol. 4, No 1.

[9] Freeman, H. (1974) 'Computer processing of line drawing images', Computer Survey, Vol.6, pp. 57-97. 
[10] Maind, M.S.B. and Wankar, M.P. (2014) 'Research Paper on Basic of Artificial Neural Network', International Journal on Recent and Innovation Trends in Computing and Communication, Volume: 2 Issue: 1, ISSN: $2321-8169,96-100$.

[11] Mashuk, M.S., Majid, M.A., Basher, N. and Rahman, T.R. 2010) 'Automatic detection of Bangla characters in Bangladeshi car registration plate', Second International Conference on Computational Intelligence, Modelling and Simulation, pp. 166-171.

[12] Ahuja, K. and Tuli, P. (2013) 'Object Recognition by Template Matching Using Correlations and Phase Angle Method', International Journal of Advanced Research in Computer and Communication Engineering Vol. 2, Issue 3.
[13] Keating, T. J., Wolf, P. R. and Scarpace, F. L. (1975) 'An Improved Method of Digital Image Correlation', Photogrammetric Engineering and Remote Sensing 41(8): 993-1002.

[14] Deb, K., Chae, H.U. and Jo, K.H. (2009) 'Vehicle License Plate Detection Method Based on Sliding Concentric Windows and Histogram', Journal of Computers, vol. 4, No 8.

[15] Saha, S., Basu, S. and Nasipuri, M. (2011) 'Automatic Localization and Recognition of License Plate Characters for Indian Vehicles', Int. J Comp Sci. Emerging Tech, vol. 2, No 4.

[16] Shidore, M. M. and Narote, S. P. (2011) 'Number Plate Recognition for Indian Vehicles', IJCSNS International Journal of Computer Science and Network Security, vol. 11 , No 2 . 